\title{
Periodic solutions for nonautonomous second order Hamiltonian systems with sublinear nonlinearity
}

\author{
Zhiyong Wang $^{1 *}$ and Jihui Zhang ${ }^{2}$
}

\section{* Correspondence: mathswzhy@126.com \\ 'Department of Mathematics, Nanjing University of Information Science and Technology, Nanjing 210044, Jiangsu, People's Republic of China \\ Full list of author information is available at the end of the article}

\section{Abstract}

Some existence and multiplicity of periodic solutions are obtained for nonautonomous second order Hamiltonian systems with sublinear nonlinearity by using the least action principle and minimax methods in critical point theory.

Mathematics Subject Classification (2000): 34C25, 37J45, 58E50.

Keywords: Control function, Periodic solutions, The least action principle, Minimax methods

\section{Introduction and main results}

Consider the second order systems

$$
\left\{\begin{array}{c}
\ddot{u}(t)=\nabla F(t, u(t)) \quad \text { a.e. } t \in[0, T], \\
u(0)-u(T)=\dot{u}(0)-\dot{u}(T)=0,
\end{array}\right.
$$

where $T>0$ and $F:[0, T] \times \mathbb{R}^{\mathbb{N}} \rightarrow \mathbb{R}$ satisfies the following assumption:

(A) $F(t, x)$ is measurable in $t$ for every $x \in \mathbb{R}^{\mathbb{N}}$ and continuously differentiable in $x$ for a.e. $t \in[0, T]$, and there exist $a \in C\left(\mathbb{R}^{+}, \mathbb{R}^{+}\right), b \in L^{1}\left(0, T ; \mathbb{R}^{+}\right)$such that

$$
|F(t, x)| \leq a(|x|) b(t), \quad|\nabla F(t, x)| \leq a(|x|) b(t)
$$

for all $x \in \mathbb{R}^{\mathbb{N}}$ and a.e. $t \in[0, T]$.

The existence of periodic solutions for problem (1.1) has been studied extensively, a lot of existence and multiplicity results have been obtained, we refer the readers to [1-13] and the reference therein. In particular, under the assumptions that the nonlinearity $\nabla F(t, x)$ is bounded, that is, there exists $p(t) \in L^{1}\left(0, T ; \mathbb{R}^{+}\right)$such that

$$
|\nabla F(t, x)| \leq p(t)
$$

for all $x \in \mathbb{R}^{\mathbb{N}}$ and a.e. $t \in[0, T]$, and that

$$
\int_{0}^{T} F(t, x) \mathrm{d} t \rightarrow \pm \infty \quad \text { as }|x| \rightarrow+\infty,
$$

Mawhin and Willem in [3] have proved that problem (1.1) admitted a periodic solution. After that, when the nonlinearity $\nabla F(t, x)$ is sublinear, that is, there exists $f(t), g$ $(t) \in L^{1}\left(0, T ; \mathbb{R}^{+}\right)$and $\alpha \in[0,1)$ such that 


$$
|\nabla F(t, x)| \leq f(t)|x|^{\alpha}+g(t)
$$

for all $x \in \mathbb{R}^{\mathbb{N}}$ and a.e. $t \in[0, T]$, Tang in [7] have generalized the above results under the hypotheses

$$
\frac{1}{|x|^{2 \alpha}} \int_{0}^{T} F(t, x) \mathrm{d} t \rightarrow \pm \infty \quad \text { as }|x| \rightarrow+\infty .
$$

Subsequently, Meng and Tang in [13] further improved condition (1.5) with $\alpha \in(0$, 1) by using the following assumptions

$$
\begin{aligned}
& \liminf _{|x| \rightarrow+\infty} \frac{1}{|x|^{2 \alpha}} \int_{0}^{T} F(t, x) \mathrm{d} t>\frac{T}{24}\left(\int_{0}^{T} f(t) \mathrm{d} t\right)^{2}, \\
& \limsup _{|x| \rightarrow+\infty} \frac{1}{|x|^{2 \alpha}} \int_{0}^{T} F(t, x) \mathrm{d} t<-\frac{T}{8}\left(\int_{0}^{T} f(t) \mathrm{d} t\right)^{2} .
\end{aligned}
$$

Recently, authors in [14] investigated the existence of periodic solutions for the second order nonautonomous Hamiltonian systems with $p$-Laplacian, here $p>1$, it is assumed that the nonlinearity $\nabla F(t, x)$ may grow slightly slower than $|x|^{p-1}$, a typical example with $p=2$ is

$$
\nabla F(t, x)=\frac{t|x|}{\ln \left(100+|x|^{2}\right)}
$$

solutions are found as saddle points to the corresponding action functional. Furthermore, authors in [12] have extended the ideas of [14], replacing in assumptions (1.4) and (1.5) the term $|x|$ with a more general function $h(|x|)$, which generalized the results of $[3,7,10,11]$. Concretely speaking, it is assumed that there exist $f(t), g(t) \in L^{1}$ $\left(0, T ; \mathbb{R}^{+}\right)$and a nonnegative function $h \in C([0,+\infty),[0,+\infty))$ such that

$$
|\nabla F(t, x)| \leq f(t) h(|x|)+g(t)
$$

for all $x \in \mathbb{R}^{\mathbb{N}}$ and a.e. $t \in[0, T]$, and that

$$
\frac{1}{h^{2}(|x|)} \int_{0}^{T} F(t, x) \mathrm{d} t \rightarrow \pm \infty \quad \text { as }-x \mid \rightarrow+\infty,
$$

where $h$ be a control function with the properties:
(a) $h(s) \leq h(t)$
(b) $h(s+t) \leq C^{*}(h(s)+h(t))$
$\forall s \leq t, s, t \in[0,+\infty)$
(c) $0 \leq h(t) \leq K_{1} t^{\alpha}+K_{2}$
$\forall s, t \in[0,+\infty)$,
(d) $h(t) \rightarrow+\infty$
$\forall t \in[0,+\infty)$,
as $t \rightarrow+\infty$,

if $\alpha=0, h(t)$ only need to satisfy conditions (a)-(c), here $C^{*}, K_{1}$ and $K_{2}$ are positive constants. Moreover, $\alpha \in[0,1)$ is posed. Under these assumptions, periodic solutions of problem (1.1) are obtained. In addition, if the nonlinearity $\nabla F(t, x)$ grows more faster at infinity with the rate like $\frac{|x|}{\ln \left(100+|x|^{2}\right)}, f(t)$ satisfies some certain restrictions and 
$\alpha$ is required in a more wider range, say, $\alpha \in[0,1]$, periodic solutions have also been established in [12] by minimax methods.

An interesting question naturally arises: Is it possible to handle both the case such as (1.8) and some cases like (1.4), (1.5), in which only $f(t) \in L^{1}\left(0, T ; \mathbb{R}^{+}\right)$and $\alpha \in[0,1)$ ? In this paper, we will focus on this problem.

We now state our main results.

Theorem 1.1. Suppose that $F$ satisfies assumption $(A)$ and the following conditions:

$\left(S_{1}\right)$ There exist constants $C \geq 0, C^{*}>0$ and a positive function $h \in C\left(\mathbb{R}^{+}, \mathbb{R}^{+}\right)$with the properties:
(i) $h(s) \leq h(t)+C$
(ii) $h(s+t) \leq C^{*}(h(s)+h(t))$
$\forall s \leq t, s, t \in \mathbb{R}^{+}$,
(iii) $\operatorname{th}(t)-2 H(t) \rightarrow-\infty$
$\forall s, t \in \mathbb{R}^{+}$,
(iv) $\frac{H(t)}{t^{2}} \rightarrow 0$
as $t \rightarrow+\infty$,
as $t \rightarrow+\infty$,

where $H(t):=\int_{0}^{t} h(s) d s$. Moreover, there exist $f \in L^{1}\left(0, T ; \mathbb{R}^{+}\right)$and $g \in L^{1}\left(0, T ; \mathbb{R}^{+}\right)$ such that

$$
|\nabla F(t, x)| \leq f(t) h(|x|)+g(t)
$$

for all $x \in \mathbb{R}^{\mathbb{N}}$ and a.e. $t \in[0, T]$;

$\left(S_{2}\right)$ There exists a positive function $h \in C\left(\mathbb{R}^{+}, \mathbb{R}^{+}\right)$which satisfies the conditions (i)(iv) and

$$
\liminf _{|x| \rightarrow+\infty} \frac{1}{H(|x|)} \int_{0}^{T} F(t, x) \mathrm{d} t>0 .
$$

Then, problem (1.1) has at least one solution which minimizes the functional $\phi$ given by

$$
\varphi(u):=\frac{1}{2} \int_{0}^{T}|\dot{u}(t)|^{2} \mathrm{~d} t+\int_{0}^{T}[F(t, u(t))-F(t, 0)] \mathrm{d} t
$$

on the Hilbert space $H_{T}^{1}$ defined by

$$
H_{T}^{1}:=\left\{u:[0, T] \rightarrow \mathbb{R}^{\mathbb{N}} \mid u \text { is absolutely continuous }, u(0)=u(T), \dot{u} \in L^{2}\left(0, T ; \mathbb{R}^{\mathbb{N}}\right)\right\}
$$

with the norm

$$
\|u\|:=\left(\int_{0}^{T}|u(t)|^{2} \mathrm{~d} t+\int_{0}^{T}|\dot{u}(t)|^{2} \mathrm{~d} t\right)^{1 / 2} .
$$

Theorem 1.2. Suppose that $\left(S_{1}\right)$ and assumption $(A)$ hold. Assume that

$$
\left(S_{3}\right) \quad \limsup _{|x| \rightarrow+\infty} \frac{1}{H(|x|)} \int_{0}^{T} F(t, x) \mathrm{d} t<0 .
$$

Then, problem (1.1) has at least one solution in $H_{T}^{1}$.

Theorem 1.3. Suppose that $\left(S_{1}\right),\left(S_{3}\right)$ and assumption $(A)$ hold. Assume that there exist $\delta>0, \varepsilon>0$ and an integer $k>0$ such that 


$$
-\frac{1}{2}(k+1)^{2} \omega^{2}|x|^{2} \leq F(t, x)-F(t, 0)
$$

for all $x \in \mathbb{R}^{\mathbb{N}}$ and a.e. $t \in[0, T]$, and

$$
F(t, x)-F(t, 0) \leq-\frac{1}{2} k^{2} \omega^{2}(1+\varepsilon)|x|^{2}
$$

for all $|x| \leq \delta$ and a.e. $t \in[0, T]$, where $\omega=\frac{2 \pi}{T}$. Then, problem (1.1) has at least two distinct solutions in $H_{T}^{1}$.

Theorem 1.4. Suppose that $\left(S_{1}\right),\left(S_{2}\right)$ and assumption $(A)$ hold. Assume that there exist $\delta>0, \varepsilon>0$ and an integer $k \geq 0$ such that

$$
-\frac{1}{2}(k+1)^{2} \omega^{2}|x|^{2} \leq F(t, x)-F(t, 0) \leq-\frac{1}{2} k^{2} \omega^{2}|x|^{2}
$$

for all $|x| \leq \delta$ and a.e. $t \in[0, T]$. Then, problem (1.1) has at least three distinct solutions in $H_{T}^{1}$.

\section{Remark 1.1.}

(i) Let $\alpha \in[0,1)$, in Theorems 1.1-1.4, $\nabla F(t, x)$ does not need to be controlled by | $\left.x\right|^{2 \alpha}$ at infinity; in particular, we can not only deal with the case in which $\nabla F(t, x)$ grows slightly faster than $|x|^{2 \alpha}$ at infinity, such as the example (1.8), but also we can treat the cases like (1.4), (1.5).

(ii) Compared with [12], we remove the restriction on the function $f(t)$ as well as the restriction on the range of $\alpha \in[0,1]$ when we are concerned with the cases like (1.8).

(iii) Here, we point out that introducing the control function $h(t)$ has also been used in [12,14], however, these control functions are different from ours because of the distinct characters of $h(t)$.

Remark 1.2. From (i) of $\left(S_{1}\right)$, we see that, nonincreasing control functions $h(t)$ can be permitted. With respect to the detailed example on this assertion, one can see Example 4.3 of Section 4.

Remark 1.3. There are functions $F(t, x)$ satisfying our theorems and not satisfying the results in [1-14]. For example, consider function

$$
F(t, x)=f(t) \frac{|x|^{2}}{\ln \left(100+|x|^{2}\right)}
$$

where $f(t) \in L^{1}\left(0, T ; \mathbb{R}^{+}\right)$and $f(t)>0$ for a.e. $t \in[0, T]$. It is apparent that

$$
|\nabla F(t, x)| \leq 4 f(t) \frac{|x|}{\ln \left(100+|x|^{2}\right)}
$$

for all $x \in \mathbb{R}^{\mathbb{N}}$ and $t \in[0, T]$. (1.12) shows that (1.4) does not hold for any $\alpha \in[0$, 1 ), moreover, note $f(t)$ only belongs to $L^{1}\left(0, T ; \mathbb{R}^{+}\right)$and no further requirements on the upper bound of $\int_{0}^{T} f(t) \mathrm{d} t$ are posed, then the approach of [12] cannot be repeated. This example cannot be solved by earlier results, such as [1-13].

On the other hand, take $h(t)=\frac{t}{\ln \left(100+t^{2}\right)}, H(t)=\int_{0}^{t} \frac{s}{\ln \left(100+s^{2}\right)} \mathrm{d} s, C=0, C^{*}=1$, then by simple computation, one has 
(i) $h(s) \leq h(t)$

(ii) $h(s+t)=\frac{s+t}{\ln \left(100+(s+t)^{2}\right)} \leq h(s)+h(t)$

$\forall s \leq t, s, t \in \mathbb{R}^{+}$,

(iii) $\operatorname{th}(t)-2 H(t)=\frac{t^{2}}{\ln \left(100+t^{2}\right)}-2 \int_{0}^{t} \frac{1}{\ln \left(100+s^{2}\right)} \mathrm{d}\left(\frac{1}{2} s^{2}\right)$

$$
=-\int_{0}^{t} \frac{2 s^{3}}{\left(100+s^{2}\right) \ln ^{2}\left(100+s^{2}\right)} \mathrm{d} s
$$

(iv) $\frac{H(t)}{t^{2}}=\frac{\int_{0}^{t} \frac{s}{\ln \left(100+s^{2}\right)} \mathrm{d} s}{t^{2}} \rightarrow 0$

$\forall s, t \in \mathbb{R}^{+}$,

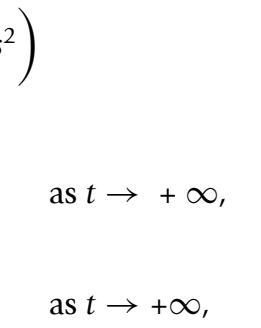

as $t \rightarrow+\infty$,

and

$$
\frac{1}{H(|x|)} \int_{0}^{T} F(t, x) \mathrm{d} t=2 \int_{0}^{T} f(t) \mathrm{d} t>0 \quad \text { as }|x| \rightarrow+\infty .
$$

Hence, $\left(S_{1}\right)$ and $\left(S_{2}\right)$ are hold, by Theorem 1.1, problem (1.1) has at least one solution which minimizes the functional $\phi$ in $H_{T}^{1}$.

What's more, Theorem 1.1 can also deal with some cases which satisfy the conditions (1.4) and (1.5). For instance, consider function

$$
F(t, x)=(0.6 T-t)|x|^{\frac{3}{2}}+(q(t), x),
$$

where $q(t) \in L^{1}\left(0, T ; \mathbb{R}^{\mathbb{N}}\right)$. It is not difficult to see that

$$
|\nabla F(t, x)| \leq \frac{3}{2}|0.6 T-t||x|^{\frac{1}{2}}+|q(t)|
$$

for all $x \in \mathbb{R}^{\mathbb{N}}$ and a.e. $t \in[0, T]$. Choose $h(t)=t^{\frac{1}{2}}, H(t)=\frac{2}{3} t^{\frac{3}{2}}, C=0, C^{*}=1$, $f(t)=\frac{3}{2}|0.6 T-t|$ and $g(t)=|q(t)|$, then $\left(S_{1}\right)$ and $\left(S_{2}\right)$ hold, by Theorem 1.1, problem (1.1) has at least one solution which minimizes the functional $\phi$ in $H_{T}^{1}$. However, we can find that the results of [14] cannot cover this case. More examples are drawn in Section 4.

Our paper is organized as follows. In Section 2, we collect some notations and give a result regrading properties of control function $h(t)$. In Section 3, we are devote to the proofs of main theorems. Finally, we will give some examples to illustrate our results in Section 4.

\section{Preliminaries}

For $u \in H_{T}^{1}$, let $\bar{u}:=\frac{1}{T} \int_{0}^{T} u(t) \mathrm{d} t$ and $\tilde{u}(t):=u(t)-\bar{u}$, then one has

$$
\|\tilde{u}\|_{\infty}^{2} \leq \frac{T}{12} \int_{0}^{T}|\dot{u}(t)|^{2} \mathrm{~d} t \quad \text { (Sobolev's inequality) }
$$

and

$$
\int_{0}^{T}|\tilde{u}(t)|^{2} \mathrm{~d} t \leq \frac{T^{2}}{4 \pi^{2}} \int_{0}^{T}|\dot{u}(t)|^{2} \mathrm{~d} t \quad \text { (Wirtinger's inequality) }
$$

where $\|\tilde{u}\|_{\infty}:=\max _{0 \leq t \leq T}|\tilde{u}(t)|$. 
It follows from assumption (A) that the corresponding function $\phi$ on $H_{T}^{1}$ given by

$$
\varphi(u):=\frac{1}{2} \int_{0}^{T}|\dot{u}(t)|^{2} \mathrm{~d} t+\int_{0}^{T}[F(t, u(t))-F(t, 0)] \mathrm{d} t
$$

is continuously differentiable and weakly lower semi-continuous on $H_{T}^{1}$ (see[2]). Moreover, one has

$$
\left(\varphi^{\prime}(u), v\right)=\int_{0}^{T}(\dot{u}(t), \dot{v}(t)) \mathrm{d} t+\int_{0}^{T}(\nabla F(t, u(t)), v(t)) \mathrm{d} t
$$

for all $u, v \in H_{T}^{1}$. It is well known that the solutions of problem (1.1) correspond to the critical point of $\phi$.

In order to prove our main theorems, we prepare the following auxiliary result, which will be used frequently later on.

Lemma 2.1. Suppose that there exists a positive function $h$ which satisfies the conditions (i), (iii), (iv) of $\left(S_{1}\right)$, then we have the following estimates:

$$
\begin{array}{ll}
\text { (1) } 0<h(t) \leq \varepsilon t+C_{0} & \text { for any } \varepsilon>0, C_{0}>0, t \in \mathbb{R}^{+}, \\
\text {(2) } \frac{h^{2}(t)}{H(t)} \rightarrow 0 & \text { as } t \rightarrow+\infty, \\
\text { (3) } H(t) \rightarrow+\infty & \text { as } t \rightarrow+\infty .
\end{array}
$$

Proof. It follows from (iv) of $\left(S_{1}\right)$ that, for any $\varepsilon>0$, there exists $M_{1}>0$ such that

$$
H(t) \leq \varepsilon t^{2} \quad \forall t \geq M_{1} .
$$

By (iii) of $\left(S_{1}\right)$, there exists $M_{2}>0$ such that

$$
\operatorname{th}(t)-2 H(t) \leq 0 \quad \forall t \geq M_{2}
$$

which implies that

$$
h(t) \leq \frac{2 H(t)}{t} \leq \varepsilon t \quad \forall t \geq M,
$$

where $M:=\max \left\{M_{1}, M_{2}\right\}$. Hence, we obtain

$$
h(t) \leq \varepsilon t+h(M)+C
$$

for all $t>0$ by (i) of $\left(S_{1}\right)$. Obviously, $h(t)$ satisfies (1) due to the definition of $h(t)$ and (2.4).

Next, we come to check condition (2). Recalling the property (iv) of $\left(S_{1}\right)$ and (2.2), we get

$$
0<\frac{h^{2}(t)}{H(t)}=\frac{h^{2}(t)}{H^{2}(t)} \cdot H(t) \leq\left(\frac{2}{t}\right)^{2} \cdot H(t)=4 \cdot \frac{H(t)}{t^{2}} \rightarrow 0 \text { as } t \rightarrow+\infty .
$$

Therefore, condition (2) holds.

Finally, we show that (3) is also true. By (iii) of $\left(S_{1}\right)$, one arrives at, for every $\beta>0$, there exists $M_{3}>0$ such that

$$
\operatorname{th}(t)-2 H(t) \leq-2 \beta \quad \forall t \geq M_{3} .
$$

Let $\theta \geq 1$, using (2.5) and integrating the relation

$$
\frac{\mathrm{d}}{\mathrm{d} \theta}\left[\frac{H(\theta t)}{\theta^{2}}\right]=\frac{\theta t \cdot h(\theta t)-2 H(\theta t)}{\theta^{3}} \leq \frac{-2 \beta}{\theta^{3}}
$$


over an interval $[1, S] \subset[1,+\infty)$, we obtain

$$
\frac{H(S t)}{S^{2}}-H(t) \leq \beta\left[\frac{1}{S^{2}}-1\right] .
$$

Thus, since $\lim _{S \rightarrow+\infty} \frac{H(S t)}{S^{2}}=0$ by (iv) of $\left(S_{1}\right)$, one has

$$
H(t) \geq \beta
$$

for all $t \geq M_{3}$. That is,

$$
H(t) \rightarrow+\infty \quad \text { as } t \rightarrow+\infty,
$$

which completes the proof. $\square$

\section{Proof of main results}

For the sake of convenience, we will denote various positive constants as $C_{i}, i=1,2$, $3, \ldots$. Now, we are ready to proof our main results.

Proof of Theorem 1.1. For $u \in H_{T}^{1}$, it follows from $\left(S_{1}\right)$, Lemma 2.1 and Sobolev's inequality that

$$
\begin{aligned}
& \left|\int_{0}^{T}[F(t, u(t))-F(t, \bar{u})] \mathrm{d} t\right| \\
& =\left|\int_{0}^{T} \int_{0}^{1}(\nabla F(t, \bar{u}+s \tilde{u}(t)), \tilde{u}(t)) \mathrm{d} s \mathrm{~d} t\right| \\
& \leq \int_{0}^{T} \int_{0}^{1} f(t) h(|\bar{u}+s \tilde{u}(t)|)|\tilde{u}(t)| \mathrm{d} s \mathrm{~d} t+\int_{0}^{T} \int_{0}^{1} g(t)|\tilde{u}(t)| \mathrm{d} s \mathrm{~d} t \\
& \leq \int_{0}^{T} \int_{0}^{1} f(t)[h(|\bar{u}|+|\tilde{u}(t)|)+C]|\tilde{u}(t)| \mathrm{d} s \mathrm{~d} t+\|\tilde{u}\|_{\infty} \int_{0}^{T} g(t) \mathrm{d} t \\
& \leq \int_{0}^{T} \int_{0}^{1} f(t)\left[C^{*}(h(|\bar{u}|)+h(|\tilde{u}(t)|))+C\right]|\tilde{u}(t)| \mathrm{d} s \mathrm{~d} t+\|\tilde{u}\|_{\infty} \int_{0}^{T} g(t) \mathrm{d} t \\
& \leq C^{*}[h(|\bar{u}|)+h(|\tilde{u}(t)|)]\|\tilde{u}\|_{\infty} \int_{0}^{T} f(t) \mathrm{d} t+C|| \tilde{u}\left\|_{\infty} \int_{0}^{T} f(t) \mathrm{d} t+\right\| \tilde{u} \|_{\infty} \int_{0}^{T} g(t) \mathrm{d} t \\
& \leq C^{*}\left[\frac{3}{C^{*} T}\|\tilde{u}\|_{\infty}^{2}+\frac{C^{*} T}{3} h^{2}(|\bar{u}|)\left(\int_{0}^{T} f(t) \mathrm{d} t\right)^{2}\right]+\|\tilde{u}\|_{\infty} \int_{0}^{T} g(t) \mathrm{d} t \\
& +C^{*}\left[h\left(\|\tilde{u}\|_{\infty}\right)+C\right]\|\tilde{u}\|_{\infty} \int_{0}^{T} f(t) \mathrm{d} t+C\|\tilde{u}\|_{\infty} \int_{0}^{T} f(t) \mathrm{d} t \\
& \leq \frac{1}{4} \int_{0}^{T}|\dot{u}(t)|^{2} \mathrm{~d} t+C_{1} h^{2}(|\bar{u}|)+C^{*}\left[\varepsilon\|\tilde{u}\|_{\infty}+C_{0}+C\right]\|\tilde{u}\|_{\infty} \int_{0}^{T} f(t) \mathrm{d} t \\
& +C\|\tilde{u}\|_{\infty} \int_{0}^{T} f(t) \mathrm{d} t+\|\tilde{u}\|_{\infty} \int_{0}^{T} g(t) \mathrm{d} t \\
& \leq\left(\frac{1}{4}+\varepsilon C_{2}\right) \int_{0}^{T}|\dot{u}(t)|^{2} \mathrm{~d} t+C_{1} h^{2}(|\bar{u}|)+C_{3}\left(\int_{0}^{T}|\dot{u}(t)|^{2} \mathrm{~d} t\right)^{1 / 2},
\end{aligned}
$$


which implies that

$$
\begin{aligned}
\varphi(u)= & \frac{1}{2} \int_{0}^{T}|\dot{u}(t)|^{2} \mathrm{~d} t+\int_{0}^{T}[F(t, u(t))-F(t, \bar{u})] \mathrm{d} t+\int_{0}^{T} F(t, \bar{u}) \mathrm{d} t-\int_{0}^{T} F(t, 0) \mathrm{d} t \\
\geq & \left(\frac{1}{4}-\varepsilon C_{2}\right) \int_{0}^{T}|\dot{u}(t)|^{2} \mathrm{~d} t-C_{3}\left(\int_{0}^{T}|\dot{u}(t)|^{2} \mathrm{~d} t\right)^{1 / 2}-\int_{0}^{T} F(t, 0) \mathrm{d} t \\
& +H(|\bar{u}|)\left[\frac{1}{H(|\bar{u}|)} \int_{0}^{T} F(t, \bar{u}) \mathrm{d} t-C_{1} \frac{h^{2}(|\bar{u}|)}{H(|\bar{u}|)}\right] .
\end{aligned}
$$

Taking into account Lemma 2.1 and $\left(S_{2}\right)$, one has

$$
H(|\bar{u}|)\left[\frac{1}{H(|\bar{u}|)} \int_{0}^{T} F(t, \bar{u}) \mathrm{d} t-C_{1} \frac{h^{2}(|\bar{u}|)}{H(|\bar{u}|)}\right] \rightarrow+\infty \quad \text { as }-\bar{u} \mid \rightarrow+\infty .
$$

As $\|u\| \rightarrow+\infty$ if and only if $\left(|\bar{u}|^{2}+\int_{0}^{T}|\dot{u}(t)|^{2} \mathrm{~d} t\right)^{1 / 2} \rightarrow+\infty$, for $\varepsilon$ small enough, (3.2) and (3.3) deduce that

$$
\varphi(u) \rightarrow+\infty \quad \text { as }\|u\| \rightarrow+\infty .
$$

Hence, by the least action principle, problem (1.1) has at least one solution which minimizes the function $\phi$ in $H_{T}^{1}$.

Proof of Theorem 1.2. First, we prove that $\phi$ satisfies the (PS) condition. Suppose that $\left\{u_{n}\right\} \subset H_{T}^{1}$ is a (PS) sequence of $\phi$, that is, $\phi^{\prime}\left(u_{n}\right) \rightarrow 0$ as $n \rightarrow+\infty$ and $\left\{\phi\left(u_{n}\right)\right\}$ is bounded. In a way similar to the proof of Theorem 1.1, we have

$$
\begin{aligned}
& \left|\int_{0}^{T}\left(\nabla F\left(t, u_{n}(t)\right), \tilde{u}_{n}(t)\right) \mathrm{d} t\right| \\
& \leq\left(\frac{1}{4}+\varepsilon C_{2}\right) \int_{0}^{T}\left|\dot{u}_{n}(t)\right|^{2} \mathrm{~d} t+C_{1} h^{2}\left(\left|\bar{u}_{n}\right|\right)+C_{3}\left(\int_{0}^{T}\left|\dot{u}_{n}(t)\right|^{2} \mathrm{~d} t\right)^{1 / 2}
\end{aligned}
$$

for all $n$. Hence, we get

$$
\begin{aligned}
\left\|\tilde{u}_{n}\right\| & \geq\left(\varphi^{\prime}\left(u_{n}\right), \tilde{u}_{n}\right)=\int_{0}^{T}\left|\dot{u}_{n}(t)\right|^{2} \mathrm{~d} t+\int_{0}^{T}\left(\nabla F\left(t, u_{n}(t)\right), \tilde{u}_{n}(t)\right) \mathrm{d} t \\
& \geq\left(\frac{3}{4}-\varepsilon C_{2}\right) \int_{0}^{T}\left|\dot{u}_{n}(t)\right|^{2} \mathrm{~d} t-C_{1} h^{2}\left(\left|\bar{u}_{n}\right|\right)-C_{3}\left(\int_{0}^{T}\left|\dot{u}_{n}(t)\right|^{2} \mathrm{~d} t\right)^{1 / 2}
\end{aligned}
$$

for large $n$. On the other hand, it follows from Wirtinger's inequality that

$$
\left\|\tilde{u}_{n}\right\| \leq\left(\frac{T^{2}}{4 \pi^{2}}+1\right)^{1 / 2}\left(\int_{0}^{T}\left|\dot{u}_{n}(t)\right|^{2} \mathrm{~d} t\right)^{1 / 2}
$$


for all $n$. Combining (3.4) with (3.5), we obtain

$$
C_{4} h\left(\left|\bar{u}_{n}\right|\right) \geq\left(\int_{0}^{T}\left|\dot{u}_{n}(t)\right|^{2} \mathrm{~d} t\right)^{1 / 2}-C_{5}
$$

for all large $n$. By (3.1), (3.6), Lemma 2.1 and $\left(S_{3}\right)$, one has

$$
\begin{aligned}
\varphi\left(u_{n}\right)= & \frac{1}{2} \int_{0}^{T}\left|\dot{u}_{n}(t)\right|^{2} \mathrm{~d} t+\int_{0}^{T}\left[F\left(t, u_{n}(t)\right)-F\left(t, \bar{u}_{n}\right)\right] \mathrm{d} t \\
& +\int_{0}^{T} F\left(t, \bar{u}_{n}\right) \mathrm{d} t-\int_{0}^{T} F(t, 0) \mathrm{d} t \\
\leq & \left(\frac{3}{4}+\varepsilon C_{2}\right) \int_{0}^{T}\left|\dot{u}_{n}(t)\right|^{2} \mathrm{~d} t+C_{1} h^{2}\left(\left|\bar{u}_{n}\right|\right)+C_{3}\left(\int_{0}^{T}|\dot{u}(t)|^{2} \mathrm{~d} t\right)^{1 / 2} \\
& +\int_{0}^{T} F\left(t, \bar{u}_{n}\right) \mathrm{d} t-\int_{0}^{T} F(t, 0) \mathrm{d} t \\
\leq & C_{6}\left[C_{4} h\left(\left|\bar{u}_{n}\right|\right)+C_{5}\right]^{2}+C_{1} h^{2}\left(\left|\bar{u}_{n}\right|\right)+C_{3}\left[C_{4} h\left(\left|\bar{u}_{n}\right|\right)+C_{5}\right] \\
& +\int_{0}^{T} F\left(t, \bar{u}_{n}\right) \mathrm{d} t-\int_{0}^{T} F(t, 0) \mathrm{d} t \\
\leq & C_{7} h^{2}\left(\left|\bar{u}_{n}\right|\right)+C_{8} h\left(\left|\bar{u}_{n}\right|\right)+C_{9}+\int_{0}^{T} F\left(t, \bar{u}_{n}\right) \mathrm{d} t-\int_{0}^{T} F(t, 0) \mathrm{d} t \\
\leq & H\left(\left|\bar{u}_{n}\right|\right)\left[C_{7} \frac{h^{2}\left(\left|\bar{u}_{n}\right|\right)}{H\left(\left|\bar{u}_{n}\right|\right)}+C_{8} \frac{h\left(\left|\bar{u}_{n}\right|\right)}{H\left(\left|\bar{u}_{n}\right|\right)}+\frac{1}{H\left(\left|\bar{u}_{n}\right|\right)} \int_{0}^{T} F\left(t, \bar{u}_{n}\right) \mathrm{d} t\right] \\
& +C_{9}-\int_{0}^{T} F(t, 0) \mathrm{d} t \rightarrow-\infty \quad \text { as } \bar{u}_{n} \mid \rightarrow+\infty .
\end{aligned}
$$

This contradicts the boundedness of $\left\{\phi\left(u_{n}\right)\right\}$. So, $\left\{\bar{u}_{n}\right\}$ is bounded. Notice (3.6) and (1) of Lemma 2.1, hence $\left\{u_{n}\right\}$ is bounded. Arguing then as in Proposition 4.1 in [3], we conclude that the (PS) condition is satisfied.

In order to apply the saddle point theorem in $[2,3]$, we only need to verify the following conditions:

$(\phi 1) \phi(u) \rightarrow+\infty$ as $\|u\| \rightarrow+\infty$ in $\tilde{H}_{T}^{1}$, where $\tilde{H}_{T}^{1}:=\left\{u \in H_{T}^{1} \mid \bar{u}=0\right\}$,

$(\phi 2) \phi(u) \rightarrow-\infty$ as $|u(t)| \rightarrow+\infty$.

In fact, for all $u \in \tilde{H}_{T}^{1}$, by $\left(S_{1}\right)$, Sobolev's inequality and Lemma 2.1 , we have 


$$
\begin{aligned}
& \left|\int_{0}^{T}[F(t, u(t))-F(t, 0)] \mathrm{d} t\right|=\left|\int_{0}^{T} \int_{0}^{1}(\nabla F(t, \bar{u}+s u(t)), u(t)) \mathrm{d} s \mathrm{~d} t\right| \\
& \quad \leq \int_{0}^{T} f(t) h(|s u(t)|)|u(t)| \mathrm{d} t+\int_{0}^{T} g(t)|u(t)| \mathrm{d} t \\
& \quad \leq \int_{0}^{T} f(t)[h(|u(t)|)+C]|u(t)| \mathrm{d} t+\|u\|_{\infty} \int_{0}^{T} g(t) \mathrm{d} t \\
& \quad \leq \varepsilon\|u\|_{\infty}^{2} \int_{0}^{T} f(t) \mathrm{d} t+\left(C_{0}+C\right)\|u\|_{\infty} \int_{0}^{T} f(t) \mathrm{d} t+\|u\|_{\infty} \int_{0}^{T} g(t) \mathrm{d} t \\
& \quad \leq \varepsilon C_{10} \int_{0}^{T}|\dot{u}(t)|^{2} \mathrm{~d} t+C_{11}\left(\int_{0}^{T}|\dot{u}(t)|^{2} \mathrm{~d} t\right)^{1 / 2},
\end{aligned}
$$

which implies that

$$
\begin{aligned}
\varphi(u) & =\frac{1}{2} \int_{0}^{T}|\dot{u}(t)|^{2} \mathrm{~d} t+\int_{0}^{T}[F(t, u(t))-F(t, 0)] \mathrm{d} t \\
& \geq\left(\frac{1}{2}-\varepsilon C_{10}\right) \int_{0}^{T}|\dot{u}(t)|^{2} \mathrm{~d} t-C_{11}\left(\int_{0}^{T}|\dot{u}(t)|^{2} \mathrm{~d} t\right)^{1 / 2} .
\end{aligned}
$$

By Wirtinger's inequality, one has

$$
\|u\| \rightarrow+\infty \Leftrightarrow\left(\int_{0}^{T}|\dot{u}(t)|^{2} \mathrm{~d} t\right)^{1 / 2} \rightarrow+\infty \quad \text { on } \tilde{H}_{T}^{1} .
$$

Hence, for $\varepsilon$ small enough, $\left(\phi_{1}\right)$ follows from (3.7).

On the other hand, by $\left(S_{3}\right)$ and Lemma 2.1, we get

$$
\int_{0}^{T} F(t, u(t)) \mathrm{d} t \rightarrow-\infty \quad \text { as }|u(t)| \rightarrow+\infty \quad \text { in } \mathbb{R}^{\mathrm{N}},
$$

which implies that

$$
\varphi(u)=\int_{0}^{T} F(t, u(t)) \mathrm{d} t-\int_{0}^{T} F(t, 0) \mathrm{d} t \rightarrow-\infty \quad \text { as }|u(t)| \rightarrow+\infty \quad \text { in } \mathbb{R}^{\mathbb{N}} .
$$

Thus, $\left(\phi_{2}\right)$ is verified. The proof of Theorem 1.2 is completed. $\square$

Proof of Theorem 1.3. Let $E=H_{T}^{1}$,

$$
H_{k}:=\left\{\sum_{j=1}^{k}\left(a_{j} \cos j \omega t+b_{j} \sin \omega t\right) \mid a_{j}, b_{j} \in \mathbb{R}^{\mathbb{N}}, j=1,2, \ldots, k\right\}
$$

and $\psi=-\phi$. Then, $\psi \in C^{1}(E, \mathbb{R})$ satisfies the (PS) condition by the proof of Theorem 1.2. In view of Theorem 5.29 and Example 5.26 in [2], we only need to prove that 


$$
\begin{array}{ll}
\left(\psi_{1}\right) \liminf \|u\|^{-2} \psi(u)>0 & \text { as } u \rightarrow 0 \text { in } H_{k}, \\
\left(\psi_{2}\right) \psi(u) \leq 0 & \text { for all } u \text { in } H_{k}^{\perp} \text {, and } \\
\left(\psi_{3}\right) \psi(u) \rightarrow-\infty & \text { as }\|u\| \rightarrow \infty \text { in } H_{k-1}^{\perp} .
\end{array}
$$

We see that

$$
F(t, x)-F(t, 0)=\int_{0}^{1}(\nabla F(t, s x), x) \mathrm{d} s
$$

for all $x \in \mathbb{R}^{\mathbb{N}}$ and a.e. $t \in[0, T]$. By $\left(S_{1}\right)$ and Lemma 2.1, one has

$$
\begin{aligned}
F(t, x)-F(t, 0) & \leq \int_{0}^{1}(f(t) h(|s x|)+g(t), x) \mathrm{d} s \\
& \leq f(t)[h(|x|)+C]|x|+g(t)|x| \\
& \leq f(t)\left[\varepsilon|x|+C_{0}+C\right]|x|+g(t)|x| \\
& =\varepsilon f(t)|x|^{2}+\left[f(t)\left(C_{0}+C\right)+g(t)\right]|x| \leq Q(t)|x|^{3}
\end{aligned}
$$

for all $|x| \geq \delta$, a.e. $t \in[0, T]$ and some $Q(t) \in L^{1}\left(0, T ; \mathbb{R}^{+}\right)$given by

$$
Q(t):=\varepsilon f(t) \delta^{-1}+\left[f(t)\left(C_{0}+C\right)+g(t)\right] \delta^{-2} .
$$

Now, it follows from (1.10) that

$$
F(t, x)-F(t, 0) \leq-\frac{1}{2} k^{2} \omega^{2}(1+\varepsilon)|x|^{2}+Q(t)|x|^{3}
$$

for all $x \in \mathbb{R}^{\mathbb{N}}$ and a.e. $t \in[0, T]$. Hence, we obtain

$$
\begin{aligned}
\psi(u) & =-\frac{1}{2} \int_{0}^{T}|\dot{u}(t)|^{2} \mathrm{~d} t-\int_{0}^{T}[F(t, u(t))-F(t, 0)] \mathrm{d} t \\
& \geq-\frac{1}{2} \int_{0}^{T}|\dot{u}(t)|^{2} \mathrm{~d} t+\frac{1}{2} k^{2} \omega^{2}(1+\varepsilon) \int_{0}^{T}|u(t)|^{2} \mathrm{~d} t-\int_{0}^{T} Q(t)|u(t)|^{3} \mathrm{~d} t \\
& \geq \frac{1}{2} \varepsilon \int_{0}^{T}|\dot{u}(t)|^{2} \mathrm{~d} t+\frac{1}{2} k^{2} \omega^{2}(1+\varepsilon)|\bar{u}|^{2} T-\|u\|_{\infty}^{3} \int_{0}^{T} Q(t) \mathrm{d} t \\
& \geq C_{12}\|u\|^{2}-C_{13} \|\left. u\right|^{3}
\end{aligned}
$$

for all $u \in H_{k}$. Then, $\left(\psi_{1}\right)$ follows from the above inequality.

For $u \in H_{k}^{\perp}$, by (1.9), one has

$$
\psi(u) \leq-\frac{1}{2} \int_{0}^{T}|\dot{u}(t)|^{2} \mathrm{~d} t+\frac{1}{2}(k+1)^{2} \omega^{2} \int_{0}^{T}|u(t)|^{2} \mathrm{~d} t \leq 0 .
$$

So, $\left(\psi_{2}\right)$ is obtained. At last, $\left(\psi_{3}\right)$ follows from $\left(\phi_{1}\right)$ which are appeared in the proof of Theorem 1.2. Then the proof of Theorem 1.3 is completed.

Proof of Theorem 1.4. From the proof of Theorem 1.1, we know that $\phi$ is coercive which implies that $\phi$ satisfies the (PS) condition. With the similar manner to [4,7], we can get the multiplicity results, here we omit the details. $\square$ 


\section{Examples}

In this section, we give some examples to illustrate our results.

Example 4.1. Consider the function

$$
F(t, x)=\left(\frac{1}{3} T-t\right) \frac{|x|^{2}}{\ln \left(100+|x|^{2}\right)}+(d(t), x),
$$

where $d(t) \in L^{1}\left(0, T ; \mathbb{R}^{\mathbb{N}}\right)$. Let $h(t)=\frac{t}{\ln \left(100+t^{2}\right)}$, then $H(t)=\int_{0}^{t} \frac{s}{\ln \left(100+s^{2}\right)} \mathrm{d} s$, by a direct computation, $\left(S_{1}\right)$ and $\left(S_{3}\right)$ hold. Then, by Theorem 1.2, we conclude that problem (1.1) has one solution in $H_{T}^{1}$. However, as the reason of Remark 1.3, the results in [1-13] cannot be applied.

Example 4.2. Consider the function

$$
F(t, x)=\left\{\begin{array}{l}
\left(\frac{2}{3} T-t\right) \frac{|x|^{2}}{\ln \left(100+|x|^{2}\right)}+A(t)|x|+B(t), \quad|x|>1, \\
-\frac{1}{4} \omega^{2}|x|^{2}+\left(\frac{1}{2} \omega^{2}+\frac{3}{2} T-\frac{9}{4} t\right)|x|^{4}-\left(\frac{1}{4} \omega^{2}+\frac{5}{6} T-\frac{5}{4} t\right)|x|^{6},|x| \leq 1,
\end{array}\right.
$$

where $A(t), B(t)$ are suitable functions which insure assumption $(A)$ hold. Also, put $H(t)=\int_{0}^{t} \frac{s}{\ln \left(100+s^{2}\right)} \mathrm{d} s, H(t)=\int_{0}^{t} \frac{s}{\ln \left(100+s^{2}\right)} \mathrm{d} s$, we see that $\left(S_{1}\right),\left(S_{2}\right)$ and (1.11) hold. By virtue of Theorem 1.4, problem (1.1) has at least three distinct solutions in $H_{T}^{1}$.

Example 4.3. Consider the function

$$
F(t, x)=\left(\frac{2}{3} T-t\right) \ln \left(100+|x|^{2}\right) \text {. }
$$

We observe that

$$
|\nabla F(t, x)| \leq\left|\frac{2}{3} T-t\right| \frac{2|x|}{100+|x|^{2}} \leq 2\left|\frac{2}{3} T-t\right|,
$$

which means $\nabla F(t, x)$ is bounded, moreover, one has

$$
\int_{0}^{T} F(t, x) \mathrm{d} t \rightarrow+\infty \quad \text { as }|x| \rightarrow+\infty
$$

Then, by the results in $[3,7,12]$, problem (1.1) has one solution which minimizes the functional $\phi$ in $H_{T}^{1}$.

In fact, our Theorem 1.1 can also handle this case. In this situation, let $h(t)=\frac{t}{100+t^{2}}$, $H(t)=\int_{0}^{t} \frac{s}{100+s^{2}} \mathrm{~d} s$, and choose $C=2, C^{*}=1 f(t)=2\left|\frac{2}{3} T-t\right|, g(t) \equiv 0$, we infer
(i) $h(s) \leq h(t)+C$
(ii) $h(s+t)=\frac{s+t}{100+(s+t)^{2}} \leq h(s)+h(t)$ $\forall s \leq t, s, t \in \mathbb{R}^{+}$,
(iii) $\operatorname{th}(t)-2 H(t)=\frac{t^{2}}{100+t^{2}}-2\left[\frac{1}{2} \ln \left(100+t^{2}\right)-\frac{1}{2} \ln 100\right]$ as $t \rightarrow+\infty$,
(iv) $\frac{H(t)}{t^{2}}=\frac{\int_{0}^{t} \frac{s}{100+s^{2}} \mathrm{~d} s}{t^{2}} \rightarrow 0$ as $t \rightarrow+\infty$. 
and

$$
\frac{1}{H(|x|)} \int_{0}^{T} F(t, x) \mathrm{d} x>0 \quad \text { as }-x \mid \rightarrow+\infty .
$$

So, by Theorem 1.1, problem (1.1) has one solution which minimizes the functional $\phi$ in $H_{T}^{1}$.

Remark 4.1. Unlike the control functions in [12], where $h(t)$ is nondecreasing, here control function $h(t)=\frac{t}{100+t^{2}}$ is bounded but not increasing.

Example 4.4. Consider the function

$$
F(t, x)=\left(\frac{1}{3} T-t\right)|x|^{\frac{4}{3}}+(k(t), x),
$$

where $k(t) \in L^{1}\left(0, T ; \mathbb{R}^{\mathbb{N}}\right)$. It is easy to check that

$$
|\nabla F(t, x)| \leq \frac{4}{3}\left|\frac{1}{3} T-t\right||x|^{\frac{1}{3}}+|k(t)| .
$$

The above inequality leads to (1.4) hold with

$$
f(t)=\frac{4}{3}\left|\frac{1}{3} T-t\right|, g(t)=|k(t)| .
$$

Take $\alpha=\frac{1}{3}$, then

$$
\frac{1}{|x|^{2 \alpha}} \int_{0}^{T} F(t, x) \mathrm{d} t \rightarrow-\infty \quad \text { as }|x| \rightarrow+\infty .
$$

So, by the theorems in $[3,7,12,13]$, problem $(1.1)$ has at least one solution in $H_{T}^{1}$. Indeed, our Theorem 1.2 can also deal with this case. Let $h(t)=t^{\frac{1}{3}}, H(t)=\frac{3}{4} t^{\frac{4}{3}}$, and choose $C=0, C^{*}=1, f(t)=\frac{4}{3}\left|\frac{4}{3} T-t\right|, g(t)=|k(t)|$, we know

$$
\begin{array}{ll}
\text { (i) } h(s) \leq h(t) & \forall s \leq t, s, t \in \mathbb{R}^{+}, \\
\text {(ii) } h(s+t)=(s+t)^{\frac{1}{3}} \leq 8(h(s)+h(t)) & \forall s, t \in \mathbb{R}^{+}, \\
\text {(iii) } t h(t)-2 H(t)=-\frac{1}{2} t^{\frac{4}{3}} \rightarrow-\infty & \text { as } t \rightarrow+\infty, \\
\text { (iv) } \frac{H(t)}{t^{2}}=\frac{3}{4 t^{\frac{2}{3}}} \rightarrow 0 & \text { as } t \rightarrow+\infty .
\end{array}
$$

Furthermore, one has

$$
\frac{1}{H(|x|)} \int_{0}^{T} F(t, x) \mathrm{d} x<0 \quad \text { as }|x| \rightarrow+\infty .
$$

Hence, $\left(S_{1}\right)$ and $\left(S_{3}\right)$ are true, by Theorem 1.2, problem (1.1) has at least one solution in $H_{T}^{1}$. However, we can find that the results in [14] cannot deal with this case.

\section{Acknowledgements}

The authors would like to thank Professor Huicheng Yin for his help and many valuable discussions, and the first author takes the opportunity to thank Professor Xiangsheng $\mathrm{Xu}$ and the members at Department of Mathematics and Statistics at Mississippi State University for their warm hospitality and kindness. This Project is Supported by National Natural Science Foundation of China (Grant No. 11026213, 10871096), Natural Science Foundation of the Jiangsu 
Higher Education Institutions (Grant No. 10KJB1 10006) and Foundation of Nanjing University of Information Science and Technology (Grant No. 20080280).

\section{Author details}

'Department of Mathematics, Nanjing University of Information Science and Technology, Nanjing 210044, Jiangsu, People's Republic of China ${ }^{2}$ Jiangsu Key Laboratory for NSLSCS, School of Mathematics Sciences, Nanjing Normal University, Nanjing 210097, Jiangsu, People's Republic of China

\section{Authors' contributions}

All authors typed, read and approved the final manuscript.

\section{Competing interests}

The authors declare that they have no competing interests.

Received: 14 May 2011 Accepted: 13 September 2011 Published: 13 September 2011

\section{References}

1. Berger, MS, Schechter, M: On the solvability of semilinear gradient operators. Adv Math. 25, 97-132 (1977). doi:10.1016/ 0001-8708(77)90001-9

2. Rabinowitz, PH: Minimax methods in critical point theory with applications to differential equations. In CBMS Reg Conf Ser in Math, vol. 65,Providence, Rl: American Mathematical Society (1986)

3. Mawhin, J, Willem, M: Critical Point Theory and Hamiltonian Systems. New York: Springer-Verlag (1989)

4. Brezis, H, Nirenberg, L: Remarks on finding critical points. Comm Pure Appl Math. 44, 939-963 (1991). doi:10.1002/ cpa.3160440808

5. Long, YM: Nonlinear oscillations for classical Hamiltonian systems with bi-even subquadratic potentials. Nonlinear Anal. 24, 1665-1671 (1995). doi:10.1016/0362-546X(94)00227-9

6. Tang, CL: Periodic solutions for non-autonomous second order systems. J Math Anal Appl. 202, 465-469 (1996). doi:10.1006/jmaa.1996.0327

7. Tang, CL: Periodic solutions for nonautonomous second order systems with sublinear nonlinearity. Proc Amer Math Soc. 126, 3263-3270 (1998). doi:10.1090/50002-9939-98-04706-6

8. Tang, CL, Wu, XP: Periodic solutions for second order systems with not uniformly coercive potential. J Math Anal Appl. 259, 386-397 (2001). doi:10.1006/jmaa.2000.7401

9. Ekeland, I, Ghoussoub, N: Certain new aspects of the calculus of variations in the large. Bull Amer Math Soc. 39, 207-265 (2002). doi:10.1090/50273-0979-02-00929-1

10. Zhao, F, Wu, X: Periodic solutions for a class of non-autonomous second order systems. J Math Anal Appl. 296, 422-434 (2004). doi:10.1016/j.jmaa.2004.01.041

11. Zhao, F, Wu, X: Existence and multiplicity of periodic solution for a class of non-autonomous second-order systems with linear nonlinearity. Nonlinear Anal. 60, 325-335 (2005)

12. Wang, Z, Zhang, J: Periodic solutions of a class of second order non-autonomous Hamiltonian systems. Nonlinear Anal. 72, 4480-4487 (2010). doi:10.1016/j.na.2010.02.023

13. Meng, Q, Tang, XH: Solutions of a second-order Hamiltonian with periodic boundary conditions. Comm Pure Appl Anal. 9, 1053-1067 (2010)

14. Wang, Z, Zhang, J: Periodic solutions of non-autonomous second order Hamiltonian systems with $p$-Laplacian. Election J Differ Equ. 2009, 1-12 (2009)

doi:10.1186/1687-2770-2011-23

Cite this article as: Wang and Zhang: Periodic solutions for nonautonomous second order Hamiltonian systems with sublinear nonlinearity. Boundary Value Problems 2011 2011:23.

\section{Submit your manuscript to a SpringerOpen ${ }^{\odot}$ journal and benefit from:}

- Convenient online submission

- Rigorous peer review

- Immediate publication on acceptance

- Open access: articles freely available online

- High visibility within the field

- Retaining the copyright to your article

Submit your next manuscript at $\gg$ springeropen.com 